Revista Médica Sinergia

Vol. 6, Núm. 6, junio 2021, e683 https://doi.org/10.31434/rms.v6i6.683

revistamedicasinergia@gmail.com

\title{
Parálisis de Bell
}

Bell's Palsy

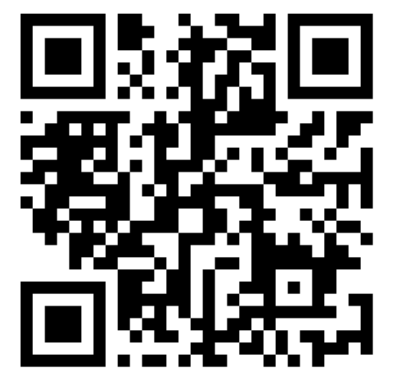

Recibido 07/08/2021
${ }^{1}$ Dra. Priscilla Montoya Alan Investigadora independiente, San José, Costa Rica

(iD https://orcid.org/0000-0002-0216-8961

${ }^{2}$ Dra. Mariel Morales Fallas Investigadora independiente, Alajuela, Costa Rica

(D) https://orcid.org/0000-0001-7151-1359

${ }^{3}$ Dra. Katherine Murillo Alvarado Investigadora independiente, San José, Costa Rica

(D) https://orcid.org/0000-0002-7156-4554

Corregido 09/09/2021
Aceptado 20/05/2021

\section{RESUMEN}

La parálisis de Bell es una afectación periférica del séptimo par craneal por una causa desconocida.

El cuadro se presenta de manera aguda y unilateral, donde se puede presentar dolor retro auricular de primera instancia y luego se presenta debilidad de los músculos faciales.

El diagnóstico es clínico, en casos especiales se utilizan estudios complementarios y posee varios diagnósticos diferenciales, entre los más importantes se encuentran el accidente cerebrovascular, neoplasia y síndrome de Ramsay Hunt.

El tratamiento se debe iniciar de forma aguda, siendo de elección los esteroides en las primeras 24 a 72 horas, asociado a terapia física. En más de un $90 \%$ de los casos con un buen pronóstico y sin secuelas a largo plazo.

PALABRAS CLAVE: parálisis; dolor; músculos; accidente cerebrovascular; esteroides.

\section{ABSTRACT}

Bell's palsy is a peripheral involvement of the seventh cranial nerve due to an unknown cause. The picture is acute and unilateral, where first instance retro atrial pain may occur and then there is weakness of the facial muscles.

The diagnosis is clinical, in special cases complementary studies are used and has several differential diagnoses, among the most important are cerebrovascular accident, neoplasia and Ramsay Hunt syndrome. 
Treatment should be started acutely, with steroids being the preferred choice in the first 24 to 72 hours, associated with physical therapy. In more than $90 \%$ of cases with a good prognosis and without long-term sequelae.

KEYWORDS: paralysis; pain; muscles; stroke; steroids.

\footnotetext{
${ }^{1}$ Médica general, graduada de la Universidad de Ciencias Médicas (UCIMED). Cód. MED13645. Correo: priella86@gmail.com

${ }^{2}$ Médica general, graduada de la Universidad de Ciencias Médicas (UCIMED). Cód. MED13964. Correo: mari mo 1@hotmail.com

${ }^{3}$ Médica general, graduada de la Universidad de Ciencias Médicas (UCIMED). Cód. MED12617. Correo: kat.murilloalvarado@gmail.com
}

\section{INTRODUCCIÓN}

Sir Francis Bell estableció a mediados del siglo XIX, la función motora del séptimo par craneal (1).

La parálisis facial periférica es una afectación del séptimo par craneal, donde se produce una disminución o ausencia total de movimiento en algunos o todos los músculos de la cara (2).

La parálisis de Bell es la forma más frecuente de parálisis facial periférica (PFP) y ocurre por una disfunción del nervio facial de etiología desconocida, aguda y unilateral (3).

Se ha documentado que tiende a ser más común en las mujeres y en algunos casos puede ser recidivante (4).

Los síntomas se instauran de una manera aguda aproximadamente de 1 a 2 días (4) y se precede usualmente de dolor medio a moderado en región retro auricular o región mastoidea (5) y posterior a esto se presenta la debilidad de los músculos faciales (6) y donde se puede apreciar el parpado caído con lagrimeo y caída de la comisura labial.

El diagnostico se basa en una adecuada historia clínica y examen físico (7) donde lo primero que se debe de valorar que sea una parálisis facial periférica (4) para posteriormente descartar los diagnostico diferenciales (3).

El tratamiento se basa tanto en el aspecto farmacológico, donde hay una fuerte evidencia que recomienda utilizar de manera temprana esteroides (8) por un periodo de 10 días y acompañado a esto comenzar desde las primeras 24 horas con la terapia física (9).

La mayoría de los pacientes presentan una resolución completa de su cuadro al mes y ya para los seis meses más de un $90 \%$ deben estar resueltas (2).

Esta revisión bibliografía tiene como objetivo una revisión del cuadro clínico, principalmente el enfoque en el tratamiento y diagnósticos diferenciales.

\section{MÉTODO}

En esta revisión se investigó y repasó el tema de parálisis de Bell con sus generalidades y se escogieron bibliografías cumpliendo con los criterios de interés entre los años 2016 hasta el 2021, entre las cuales tenemos libros de texto, artículos y revistas digitales como Elsevier, tanto en inglés como en español.

Se utilizó búsqueda entre los temas "parálisis periférica", "paralisis de Bell", "nervio facial" "causas de parálisis de Bell"; y con toda esta información que se obtuvo, se hizo un minucioso análisis y se escogió lo más relevante y de mayor importancia científica para ofrecer una revisión de calidad.

\section{GENERALIDADES}

El séptimo par craneal llamado nervio facial, tiene una función tanto sensitiva como motora. En la parte sensitiva se encarga de inervar los dos tercios anteriores de la 
lengua y en cuanto la parte motora inerva todos los músculos de la expresión facial (10).

El nervio facial sale del cráneo por el agujero estilomastoideo situado entre la apófisis estiloides antero medialmente, la fosa yugular medialmente y la apófisis mastoides postero lateralmente, sale del agujero a 21 $\mathrm{mm}$ de la superficie cutánea, después atraviesa las regiones retroestilodea y parotídea, y termina su trayecto hasta los músculos de la cara pasando bajo el sistema musculo aponeurótico superficial (SMAS) (11).

El trayecto retroestiloideo es corto, el nervio adopta una orientación anteroinferior para entrar en la región parotídea y atraviesa el diafragma estiloideo entre el vientre posterior del músculo digástrico lateralmente y el músculo estilohioideo medialmente, inervando ambos músculos y dando sus ramas terminales.

\section{ETIOLOGÍA}

La parálisis de Bell se da cuando ocurre inflamación en el canal intrapetroso más específicamente en el agujero estilomastoideo (7) sin una causa aparente, aunque se ha estudiado que puede estar relacionado al virus del herpes simple tipo 1 (4), una reactivación del virus herpes zoster, un traumatismo o tumoración en dicha área (5).

Otra causa que se ha propuesto para este tipo de parálisis es que ocurre una insuficiencia micro circulatoria del nervio por una reacción auto inmune (12).

\section{EPIDEMIOLOGÍA}

Este tipo de parálisis periférica tiene una elevada incidencia, 20-50 casos por cada 100.000 habitantes, donde la mayoría de estos pacientes se atienden en el servicio de emergencias para descartar un ictus (4), es más frecuente en mujeres y el embarazo puede ser un factor predisponente (1), además el antecedente de migraña o la ingesta de estatinas también se ha relacionado al aumento del riesgo para parálisis de Bell (8).

Como un antecedente heredo familiar, se puede encontrar en un $9 \%$ de los casos y puede ser recidivante en un $10-20 \%$ de los casos (4).

\section{MANIFESTACIONES CLÍNICAS}

Los síntomas se instauran de una forma aguda entre 1 o 2 días y usualmente esta precedido de un dolor usualmente moderado en la región retroauricular o mastoideo (1).

Entre los síntomas que pueden presentar se encuentran la disfunción motora (caída de la ceja y del párpado inferior, imposibilidad de cerrar el ojo y desviación de la boca al lado sano) (4).

Otros síntomas adicionales pueden incluir afectación del componente gustativo como pérdida de la sensación del gusto en los dos tercios anteriores de la lengua, sensación de sabor metálico en la lengua, disminución de la salivación (13); así como hiperacusia y acumulación de lágrimas en el parpado inferior por lo que pueden presentar sensación de visión borrosa.

En muchos pacientes, la parálisis se instaura durante la noche y se hace más evidente por la mañana; la notan los familiares o bien el paciente al mirarse al espejo o al tratar de ingerir algún líquido y este se derrama por la comisura bucal débil (4).

\section{DIAGNÓSTICO}

Para confirmar que se trata de una parálisis facial periférica, tanto por la historia clínica y como describe Lassaletta et al., "la anamnesis irá orientada a establecer el modo de inicio de la parálisis, brusco o progresivo, y la presencia de episodios previos. Se debe preguntar por enfermedades infecciosas recientes, 
antecedentes oncológicos, enfermedades vasculares, cardiopatías y alteraciones de la coagulación, así como la existencia de sintomatología asociada (dolor, vértigo, otorrea o hipoacusia)" (2,7-10).

El examen físico el cual se describe en la tabla 1.

Excluir otras causas: en la práctica, si no hay ningún rasgo atípico, no se necesita realizar serologías, análisis del líquido cefalorraquídeo (LCR) o resonancia magnética (RM), ya que, las imágenes usualmente no son requeridas y quedan al criterio médico $(10,4)$.

La RM con gandolino permite objetivar la inflamación del nervio en algún punto de su trayecto $(4,12)$.

El antecedente de otitis aguda o reactivación de otitis crónica se recomienda valoración por otorrinolaringología (4).

¿Qué debemos hacer ante una parálisis de Bell bilateral?

- Es menos del 1\% de los casos.

- Según describe Lassaleta et al., "abordaje multidisciplinario para descartar la presencia de una enfermedad grave. Debe incluir una completa historia clínica y exploración física, estudios en sangre periferica (hemograma, bioquímica, pruebas tiroideas, serologías (toxoplasma, citomegalovirus, rubéola, brusella entre otros), anticuerpos (anticuerpos antinucleares, antifosfolípidos, anticuerpos anticitoplasma de neutrófilos) y de diagnóstico por la imagen (RM o LCR)" (7).

¿Qué signos nos hacen pensar que NO se trata de una parálisis de Bell?

- Describe Lassaletta et al., "Parálisis progresiva en lugar de brusca, bilateral, empeoramiento a partir de la tercera semana o tras una mejoría inicial, ausencia de recuperación en 4 meses, parálisis recurrente en el mismo lado de la cara, debilidad asimétrica en las distintas zonas de la cara o combinación de signos de flacidez y espasmo en el mismo paciente" y pensar en diagnósticos diferenciales.

\begin{tabular}{|c|c|}
\hline Examen neurológico & $\begin{array}{l}\text { - Parálisis o debilidad que afecta todos los músculos faciales de un } \\
\text { lado, incluyendo la frente y descartar patología central. } \\
\text { - Sensibilidad corneal (V Par craneal) (2). } \\
\text { - Si hay otros nervios craneales involucrados en la debilidad facial } \\
\text { no se debe a la parálisis de Bell (10). }\end{array}$ \\
\hline $\begin{array}{l}\text { Reflejo palpebral - oculogiro } \\
\text { (signo de Bell) }\end{array}$ & $\begin{array}{l}\text { Es el movimiento ascendente del ojo, cuando se realiza un intento de } \\
\text { cerrarlos forzadamente, aproximadamente un } 75 \% \text { de la gente sana } \\
\text { sin afección lo posee. }\end{array}$ \\
\hline $\begin{array}{l}\text { Prueba de sonoridad con } \\
\text { estetoscopio }\end{array}$ & $\begin{array}{l}\text { Existe dificultad para cerrar el ojo del lado paralizado; al intentar } \\
\text { cerrarlo, el globo ocular puede desviarse hacia arriba y ligeramente } \\
\text { hacia dentro (signo de Bell) }(2,7) \text {. }\end{array}$ \\
\hline Gusto & $\begin{array}{l}\text { El paciente escucha el girar de un tenedor por medio del } \\
\text { estetoscopio. Representa la hiperacusia ocasionada por la parálisis } \\
\text { del músculo estapedio, }\end{array}$ \\
\hline Conducto auditivo & Probar los sabores dulces, salados y ácidos. \\
\hline \multicolumn{2}{|c|}{ 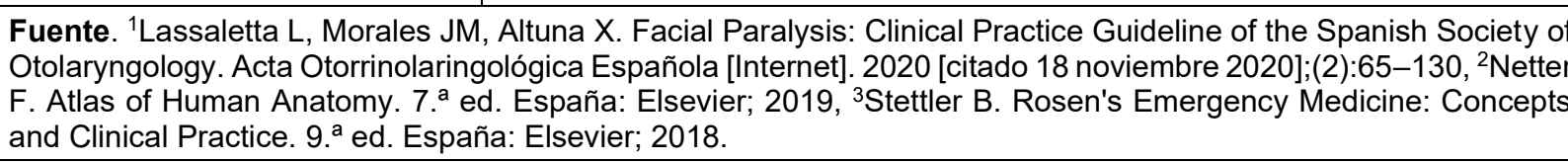 } \\
\hline
\end{tabular}




\section{DIAGNÓSTICO DIFERENCIAL}

1. Síndrome de Ramsay Hunt (10)

- Etiología: Herpes zoster en ganglio geniculado.

- Cuadro clínico: vesículas en el conducto auditivo externo (CAE) y paladar blando, pérdida de la audición y vértigo pueden ocurrir. Cuadro muy difícil de diferenciar con la parálisis de Bell.

- Pronóstico: menor que en la parálisis de Bell.

- Tratamiento: antivirales

2. Debilidad facial bilateral $(10,12)$

- Menos del 1\% de los casos.

- Causas: infecciosas (Enfermedad de Lyme), Virus de la inmune deficiencia humana, mastoidistis, difteria $y$ botulismo, sarcoidosis, trauma y tumores en la base del cráneo, entre otros.

\section{Espasmo hemifacial (10)}

- Espasmo irregular y sin dolor unilateral.

- Pacientes edad media.

- Cuadro clínico: inicia en el músculo orbicular de los ojos, y progresa gradualmente sobre los años y afecta otros músculos faciales del mismo lado.

- Varía de leve a severo.

4. Mioquimia del músculo opercular de los ojos

- Es una contracción nerviosa irritante, usualmente del párpado inferior (10).

5. Otitis media aguda (7)

- Inflamación del odio medio junto con al menos una de las siguientes: otalgia, otorrea, cefalea, infección de vías aéreas superiores.

- Tratamiento. Antibioticoterapia.

\section{Síndrome de Guillan Barré}

- En estos casos, la parálisis suele ser bilateral (7).

7. Accidente cerebro vascular (7)

- Músculos de la frente usualmente sin afección.

- Las extremidades de un lado del cuerpo también involucradas.

8. Neoplasia (7)

- Schwannoma vestibular (neuroma acústico).

- Tumor benigno originado de la rama del séptimo nervio craneal.

- Clínica: tinnitus e hipoacusia por compresión nerviosa. Así como, vértigo periférico, cefalea o ambas.

- Diferenciarlo con historia clínica, examen físico e imágenes.

9. Tics y disquinesia tardía

- Involucran a los músculos perfórales $y$ faciales (10).

10. Blefarospasmo.

- Una disponía focal que afecta al músculo opercular de los ojos (10).

\section{TRATAMIENTO}

\section{Objetivo}

- Recuperar la función del músculo

- Evitar daño a la córnea.

En la tabla 2, se describen las diferentes opciones terapéuticas.

Posterior a que se recibe el tratamiento inicial, para poder precisar el grado de recuperación o de secuelas que va a tener el paciente se utiliza la escala de HouseBrakmann. (ver tabla 3) (15).

\section{Casos especiales}

\section{Embarazadas}

- Monitorear por preeclampsia (7). 
Tabla 2. Opciones terapéuticas de la parálisis de Bell

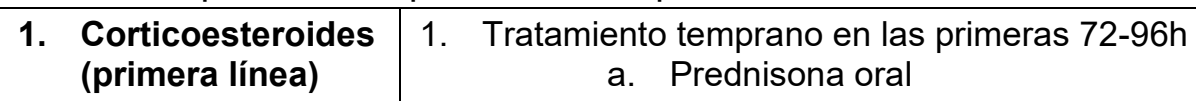
(primera línea)

i. Adultos

1. Primera opción. Prednisona $60 \mathrm{mg} / \mathrm{d}$ por 5 días, seguido por $25 \mathrm{mg}$ dos veces/d por 10 días.

2. Segunda opción. $410 \mathrm{mg}$ administrados en forma descendente por 10 días.

3. Tercera opción. Prednisona ajustada a 1 $\mathrm{mg} / \mathrm{kg} / \mathrm{día}$ durante 5 días con reducción progresiva de $10 \mathrm{mg} /$ día los días sucesivos hasta llegar a cero. Un ejemplo demostrado con un paciente de $75 \mathrm{~kg}$ :

Dia 1 - 5: $75 \mathrm{mg}$

Dia 6: $70 \mathrm{mg}$

Día 7: $60 \mathrm{mg}$

Día 8: $50 \mathrm{mg}$

Día 9: $40 \mathrm{mg}$

Día 10: $30 \mathrm{mg}$

Día 11: $20 \mathrm{mg}$

Día 12: $10 \mathrm{mg}$

b. Prednisolona oral

Día 13: $0 \mathrm{mg}$

i. Adultos

1. Primera opción $60 \mathrm{mg} / \mathrm{d}$ por 5 días, seguido de $25 \mathrm{mg}$ a $10 \mathrm{mg}$ dos veces/d durante 10 días.

2. Deflazacort. $60 \mathrm{mg} / \mathrm{d}$ por 7 días. Luego ir reduciendo de modo progresivo de 12 en 12 mg cada 3 días hasta suspensión del tratamiento (8).

2. Preventivo $\quad$ Recomendado oclusiones oculares, gafas, lágrimas artificiales y colirios para evitar el daño de la córnea durante los días en que el paciente no ocluya el ojo $(4,7)$.

Protectores gástricos como omeprazol $20 \mathrm{mg} / \mathrm{d}$ en pacientes con antecedentes de úlceras gástricas, mayores de 60 años o tratamiento concomitante con antiagregantes plaquetarios (8).

3. Terapia física

Inicialmente junto a los esteroides. En casos de debilidad muscular persistente (4).

4. Antivirales

Antivirales. Uso beneficioso y marginal, se recomienda solo en los casos graves o cuando de sospecha (por el dolor, por ejemplo) que un agente casual puede ser el herpes zóster, aunque no haya una clara erupción de vesículas.

Valaciclovir $1 \mathrm{~g}$ cada $8 \mathrm{~h}$ por 7 días

Famciclovir $250 \mathrm{mg}$ cada $8 \mathrm{~h}$ por 7 días

Aciclovir $800 \mathrm{mg}$ cada $4 \mathrm{~h}$ por día

\section{Descompresiones quirúrgicas}

No poseen evidencia $(3,4)$.

Se describe que podría utilizarse en casos de parálisis facial persistente sea idiopática o debido a trauma (13).

Fuente. ${ }^{1}$ Lassaletta L, Morales JM, Altuna X. Facial Paralysis: Clinical Practice Guideline of the Spanish Society of Otolaryngology. Acta Otorrinolaringológica Española [Internet]. 2020 [citado 18 noviembre 2020];(2):65-130,

${ }^{2} Z$ arranz JJ. Neurologia. 6. ${ }^{a}$ ed. España: Elsevier; 2018, ${ }^{3}$ Zarranz JJ. Neurologia. 6. ${ }^{a}$ ed. España: Elsevier; 2018, ${ }^{4}$ Kliegman R. Nelson. Tratado de pediatría. 21. ${ }^{a}$ ed. España: Elsevier; 2020, ${ }^{5}$ Netter F. Atlas of Human Anatomy. 


\begin{tabular}{|l|l|}
\hline \multicolumn{2}{|l|}{ Tabla 3. Escala de House Brakmann } \\
\hline Grado I & Función normal en todos los territorios. \\
\hline Grado II: Disfunción leve & $\begin{array}{l}\text { Ligera o leve debilidad de la musculatura, en reposo } \\
\text { presenta simetría normal, no hay contracturas ni } \\
\text { espasmos faciales. }\end{array}$ \\
\hline Grado III: Disfunción moderada. & $\begin{array}{l}\text { Diferencia clara entre ambos lados de la cara, sin llegar a } \\
\text { ser desfiguradora. Incompetencia para el cierre palpebral } \\
\text { complete, hay movimiento de la región frontal y asimetría } \\
\text { de la comisura labial. En reposo simetría y tono normales. }\end{array}$ \\
\hline $\begin{array}{l}\text { Grado IV: Disfunción intermedia } \\
\text { moderada - grave }\end{array}$ & $\begin{array}{l}\text { Debilidad y/o asimetría desfiguradora. En reposo simetría } \\
\text { y tono normal. No hay movimiento de la región frontal, } \\
\text { imposibilidad para cerrar el ojo totalmente. Espasmos } \\
\text { faciales. }\end{array}$ \\
\hline $\begin{array}{l}\text { Grado V: Disfunción grave } \\
\text { Gan solo ligera actividad motora perceptible. En reposo, } \\
\text { asimetría. }\end{array}$ \\
\hline $\begin{array}{l}\text { Fuente. }{ }^{1} \text { Tobar AM, Catacolí JG, Echandia C. Evaluación del índice clínico de función del nervio facial en } \\
\text { pacientes con parálisis de Bell. Revista Colombiana de Medicina Física y Rehabilitación [Internet]. 2016 [citado 18 } \\
\text { noviembre 2020];(26) }\end{array}$ \\
\hline
\end{tabular}

- Usualmente en tercer trimestre o primera semana post parto (2).

- Los corticoesteroides son seguros al utilizarlos en el tercer semestre, sin embargo, para minimizar riesgos para el feto se recomienda utilizar prednisolona o metilprednisolona, ya que atraviesan la barrera placentaria en menor medida (2).

\section{Niños}

- Prioridad evitar complicaciones en la córnea.

- Corticoesteroides $1 \mathrm{mg} / \mathrm{kg} / \mathrm{d}$ durante 5-7 días, seguido de una pauta descendente durante una semana, con o sin antiviral (5).

- Lágrimas artificiales frecuentemente (3 a 6 veces por día) y en las noches una oclusión ocular.

- Referir a neurología a niños menores de 2 años.

\section{Diabetes mellitus}

\section{PRONÓSTICO}

En fase aguda es difícil de sentar un pronóstico, sin embargo, sí hay grado de recuperación hacia la sexta semana es un buen indicador del pronóstico final. Los pacientes que inician la recuperación entre la 4ta y 6ta semana tienen mejor pronóstico, ya que, aquellos que no la presentan probablemente mostrarán secuelas y son aproximadamente el 10\% (14).

Las secuelas no deben considerarse como definitivas antes de los 6 a 8 meses (7), único factor pronóstico seguro es si es 
incompleto, ya que estas, tiene tasas mayores del $90 \%$ de resolución. Mientras, las completas llegan a un 60\% (4).

Los factores de peor pronóstico son:

- Edad superior a los 60 años

- Diabetes mellitus e hipertensión arterial.

- Falta de terapia física $(4,7)$.

- Embarazadas (2)

En el caso particular de los niños, presentan un muy buen pronóstico y de manera natural en casi un $100 \%$ de los casos (4).

Electromiografía: ayuda a sentar el pronóstico, pero no en las primeras horas, más bien, entre el 5 to al $7 \mathrm{mo}$ día del inicio de los síntomas, ya que detectan una denegación completa el pronóstico, será peor (4).

Electroneurografía: puede ayudar en el pronóstico en pacientes con parálisis facial completa (7).

\section{COMPLICACIONES}

1. Irritación en el ojo y ulceración corneal

2. Potencialmente de largo plazo incluye:

- Obstrucción nasal

- Incompetencia oral

- Epífora o lagrimeo constante

- Disestesia, disgeusia o ageusia, por regeneración sensorial incompleta

- Sincinesia (movimiento ocular inconsistente), por incorrecta reinnervación

- Espasmos hemifacial

\section{CONCLUSIONES}

La parálisis de Bell es una patología ocasionada por la inflamación del recorrido del nervio facial extra craneal y etiología desconocida, posee una sintomatología aguda y brusca, el cuadro clínico aparte de la parálisis facial periférica unilateral, se adicionan el dolor en la región retro auricular o mastoideo, pérdida del gusto en los 2/3 anteriores e hiperacusia. El diagnóstico es clínico, el utilizar los exámenes de laboratorio, LCR o imágenes en casos donde la presentación es atípica como presentación insidiosa y bilateral, ya que se debe pensar en diagnósticos diferenciales, entre los cuales se destacan debido a su clínica, tratamiento y pronóstico el accidente cerebrovascular, síndrome Ramsay Hunt y neoplasia.

Ya con un diagnóstico certero, se recomienda el iniciar el tratamiento a no más tardar en las primeras 72 a 96 horas con corticoesteriodes donde está la mayor evidencia científica, de manera escalonada por 10 días en adultos y embarazadas en el tercer trimestre, seguido de evitar complicaciones corneales en todos los casos, principalmente en niños en donde, es de mayor valor que los esteroides, ya que, inclusive sin estos, su resolución llega casi a un $100 \%$ de los casos, junto con terapia física.

El pronóstico con un correcto diagnóstico, en la mayoría de los casos es mayor a un $90 \%$ en las primeras seis semanas y decae en casos de factores de riesgo, las secuelas motoras no deben considerarse definitivas hasta los 6 a 8 meses posterior del inicio del cuadro.

\section{Los autores declaran no tener conflicto de interés.}

\section{REFERENCIAS}

1. Aminoff $M$, Douglas V. Creasy and Resnik's Maternal-Fetal Medicine: Principles and Practice. 8. ${ }^{a}$ ed. España: Elsevier; 2019. Disponible en: https://www-clinicalkey-

es.binasss.idm.oclc.org/\#!/content/book/3-s2.0B9780323479103000668?scrollTo $=\% 23 \mathrm{hl} 000080$ 으

2. Lassaletta L, Morales JM, Altuna X. Facial Paralysis: Clinical Practice Guideline of the Spanish Society of Otolaryngology. Acta Otorrinolaringológica Española [Internet]. 2020 [citado 18 noviembre 2020];(2):65-130. 
Disponible en: https://www-clinicalkeyes.binasss.idm.oclc.org/\#!/content/journal/1-s2.0S2173573520300235

3. Louro A. Fisterra [Internet]. Elsevier. 2018 [citado 10 enero 2021]. Disponible en: https://wwwclinicalkeyes.binasss.idm.oclc.org/\#!/content/guides techni ques/52-s2.0-mt fis 339

4. Zarranz JJ. Neurologia. 6. ${ }^{a}$ ed. España: Elsevier; 2018. Disponible en: https://www-clinicalkeyes.binasss.idm.oclc.org/\#!/content/book/3-s2.0B9788491130710000088?scrollTo=\%23hl000038 $\underline{3}$

5. Kliegman R. Nelson. Tratado de pediatría. 21. ${ }^{a}$ ed. España: Elsevier; 2020. Disponible en: https://www-clinicalkeyes.binasss.idm.oclc.org/\#!/content/book/3-s2.0B978849113684200635X

6. Netter F. Atlas of Human Anatomy. 7. a ed. España: Elsevier; 2019. Disponible en: https://www-clinicalkey-

es.binasss.idm.oclc.org/\#!/content/book/3-s2.0B9780323393225000220?scrollTo $=\% 23 \mathrm{hl000193}$ $\underline{5}$

7. Bell Palsy [Internet]. Elsevier Point of Care. 2019 [citado 10 enero 2021]. Disponible en: https://www-clinicalkey-

es.binasss.idm.oclc.org/\#!/content/clinical overvi ew/67-s2.0-763cfcac-20d4-4057-8834c40150c4bf80

8. Jiménez L. Medicina de urgencias y emergencias. 6. ${ }^{a}$ ed. España: Elsevier; 2018. Disponible en : https://www-clinicalkeyes.binasss.idm.oclc.org/\#!/content/book/3-s2.0B9788491132080001583?scrollTo $=\% 23 \mathrm{hl} 000010$ $\underline{9}$

9. Stettler B. Rosen's Emergency Medicine: Concepts and Clinical Practice. 9. ${ }^{a}$ ed. España: Elsevier; 2018. Disponible en: https://wwwclinicalkey-

es.binasss.idm.oclc.org/\#!/content/book/3-s2.0B9780323354790000957? scrollTo $=\% 23 \mathrm{hl} 000036$ 1

10. Feather A, Randall D, Waterhouse M. Kumar and Clark's Clinical Medicine. 10. ${ }^{a}$ ed. España: Elsevier; 2020. Disponible en: https://wwwclinicalkeyes.binasss.idm.oclc.org/\#!/content/book/3-s2.0B9780702078682000269? scrollTo $=\% 23 \mathrm{hl} 000633$ $\underline{6}$

11. Barbut J, Tankéré F. Anatomía del nervio facial. EMC Otorrinolaringología [Internet]. 2017 [citado 18 noviembre 2020] ;(46). Disponible en: https://www-clinicalkeyes.binasss.idm.oclc.org/\#!/content/emc/51-s2.0S1632347517855132?scrollTo=\%23hl0000877

12. Mattox D, Vivas E. Cummings Otolaryngology: Head and Neck Surgery. 7. ${ }^{a}$ ed. España: Elsevier;
2020. Disponible en: https://www-clinicalkeyes.binasss.idm.oclc.org/\#!/content/book/3-s2.0B9780323611794001721?scrollTo=\%23hl000032 $\underline{0}$

13. Pasternak J, Lanier W. Stoelting's Anesthesia and Co-Existing Disease. 7. ${ }^{a}$ ed. España: Elsevier; 2018. Disponible en: https://www-clinicalkeyes.binasss.idm.oclc.org/\#!/content/book/3-s2.0B9780323401371000156? scrollTo $=\% 23 \mathrm{hl} \mid 000046$ 으

14. Smith G, Shy M. Goldman-Cecil Medicine. 26. ${ }^{a}$ ed. España: Elsevier; 2020. Disponible en: https://www-clinicalkeyes.binasss.idm.oclc.org/\#!/content/book/3-s2.0B9780323532662003921?scrollTo=\%23hl000138 $\underline{2}$

15. Tobar AM, Catacolí JG, Echandia C. Evaluación del índice clínico de función del nervio facial en pacientes con parálisis de Bell. Revista Colombiana de Medicina Física y Rehabilitación [Internet]. 2016 [citado 18 noviembre 2020];(26). Disponible en: https://revistacmfr.org/index.php/rcmfr/article/dow $\underline{\text { nload } / 163 / 158}$ 